
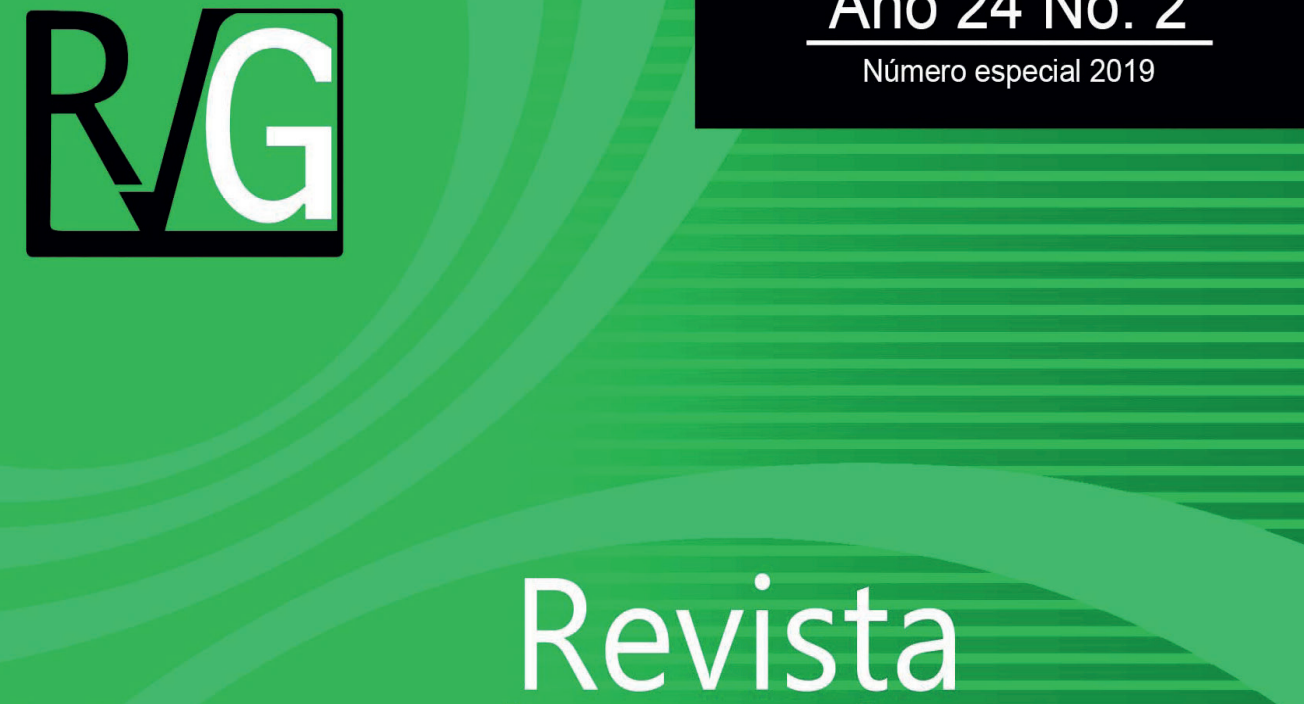

Venezolana de

$\frac{1}{6}$

$\frac{\mathbb{O}}{\frac{1}{0}}$
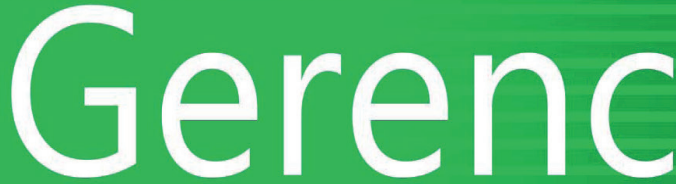

0

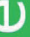

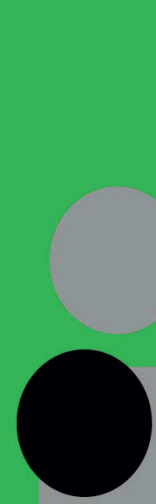

ro

u

$>$

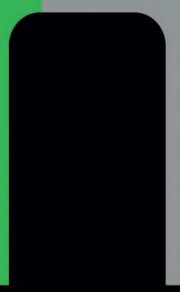




\title{
Modelos de mercadeo relacional en educación: articulación con el docente universitario
}

\author{
Gómez-Bayona, Ledy ${ }^{1}$ \\ Ferre-Pavia, Carme ${ }^{2}$ \\ Arrubla Zapata, Juan Pablo ${ }^{3}$
}

\section{Resumen}

En los últimos tiempos, fenómenos como la globalización han hecho que en el contexto educativo se construyan nuevas maneras de relacionarse para generar valor en la percepción de cada institución. Es por ello que, a partir de esta investigación se pretende identificar los modelos de mercadeo relacional que se han aplicado en algunas Instituciones de Educación Superior para comprender cuáles son sus principales variables de análisis y de qué manera han involucrado al docente. Para lograrlo, se emplea la metodología de revisión de literatura y como resultado se tienen dieciocho modelos de mercadeo con enfoque relacional, concluyendo que dichos modelos se han centrado en diferentes públicos como estudiantes, egresados y la industria, pero la aplicación a los docentes como sujeto del modelo ha sido escasa.

Palabras clave: mercadeo relacional; Gestión educativa; Docente universitario; Reputación.

\footnotetext{
Recibido: 16-08-19 Aceptado: 29-10-19
}

1 Docente asociado de la Facultad de Ciencias Empresariales de la Universidad de san Buenaventura Medellín-Colombia, Doctoranda en Administración de la Universidad de Medellín. ledy.gomez@usbmed.edu. co. ORDCID: 0000-0003-4122-0344

2 Profesora titular del departamento de Medios, Comunicación y Cultura de la Universidad Autónoma de Barcelona-España. Doctora en Ciencias de la Información. carme.Ferre@uab.cat. ORDCID: 0000-00027258-6376

3 Docente titular de la Facultad de Ciencias Económicas de la Universidad de Medellín - Medellín-Colombia. Doctor en Dirección y Administración de Empresas. jarrubla@udem.edu.co. ORDCID: 0000-0002-6240-3564 


\title{
Models of relational marketing in education and its articulation with the university teacher
}

\begin{abstract}
In recent times, phenomena such as globalization have led to the creation of new ways of relating in the educational context to generate value in the perception of each institution. That is why, based on this research, the aim is to identify the relational marketing models that have been applied in some Higher Education Institutions to understand what their main analysis variables are and how they have involved the teacher. To achieve this, the literature review methodology is used and as a result there are eighteen marketing models with a relational approach, concluding that they have focused on different audiences such as students, graduates and industry, but the application to teachers as the subject of the model It has been scarce.
\end{abstract}

Keywords: Relational Marketing; Educational Management; University Teacher; Reputation

\section{Introducción}

La gestión de las universidades se ha transformado en los últimos tiempos con el objetivo de estar más cerca a las necesidades de los usuarios y construir propuestas de valor que generen satisfacción y buenos rendimientos (Rojas, 2018). Es ahí donde la planeación de estrategias desde el mercadeo ha sido significativa y ha marcado un nuevo paradigma en la manera de relacionarse con la comunidad académica. El enfoque de mercadeo en la gestión administrativa se ha modificado hacia una comunicación más cercana con los diferentes públicos que alimentan el sector educativo, con el fin de construir un relacionamiento duradero (HennigThurau, Langer \& Hansen, 2001). Es entonces donde el mercadeo relacional empieza a ser funcional y a ganar un espacio dentro de las estrategias y acciones que se implementan en las universidades.

De la misma manera, aspectos como la competitividad hacen que las universidades busquen alternativas de sostenibilidad. Algunos rankings educativos como ARWU, Times Higher Education, QS Top University, World's Best Universities, Global Universities, Leiden Ranking, Webometrics Ranking of World Universities, o Ranking de el Financial Times, todos de alcance mundial, han venido considerando componentes de medición para generar visibilidad y posicionamiento de las universidades en la sociedad; eso hace que los directivos universitarios incorporen dentro de sus modelos de administración diferentes aspectos como la gestión de recursos, la contextualización del entorno, la globalización y la producción investigativa, según informa Universitas 21 (U21). Los tres primeros países en tener los mejores sistemas nacionales de 
Modelos de mercadeo racional en educación: articulación con el docente universitario Gómez-Bayona Ledy, Ferre-Pavia Carme, Arrubla Zapata Juan Pablo

educación superior en 2019 son Estados Unidos, Suiza y Reino Unido, mientras que en Iberoamérica están dentro de los tres primeros Chile, México y España. Así mismo, el Times Higher Education lanzó el ranking de impacto universitario donde se tiene en cuenta el éxito de las universidades y las clasifica dependiendo de los objetivos de desarrollo sostenible de las Naciones Unidas. En este, Nueva Zelanda, Canadá y el Reino Unido son pioneras en el Top 20 global y en el Top 5 de Latinoamérica están Chile y México.

Los anteriores rankings hacen que las universidades, en el afán de sobrevivir en un sector tan competido, hagan que los directivos construyan sus propias estrategias que les permiten generar relacionamiento $y$ posicionamiento a nivel nacional e internacional (Ramachandran, 2010). Aspectos como el liderazgo académico son significativos, ya que permiten planear propuestas que le generen beneficios a quienes tienen relación con la organización (Oplatka, 2018). En el caso particular del mercadeo educativo, las áreas encargadas de gestionar el mercadeo institucional se han venido transformando con el objetivo de incluir una orientación de mercado que articule los individuos a los objetivos que se pretenden alcanzar (Oplatka, HemsleyBrown, 2007; Kalenskaya, Gafurov \& Novenkova, 2013). Así, el cambio del paradigma del mercadeo transaccional al relacional ha ganado espacio en el sector educativo, impulsando que se construya una nueva manera de hacer mercadeo, donde los lineamientos desde los directivos y el apoyo de los colaboradores estén en sintonía con las necesidades de la sociedad. Es entonces cuando se empiezan a implementar estrategias de mercadeo de manera estratégica y táctica, con fundamentación teórica y estructurada.

Sin embargo, la literatura es incipiente y requiere mayor exploración en diferentes contextos (Oplatka, 2006; Csikosova, 2014). En Iberoamérica se cuenta con algunos estudios que han facilitado la comprensión de algunas variables de mercadeo desde el aspecto comercial (Bennett \& Ali-Choudhury, 2009), de la satisfacción de los públicos (Amoli \& Aghashahi, 2016). Si bien es cierto que las anteriores investigaciones han aportado significativamente a las características del mercadeo relacional en componentes como confianza, satisfacción, lealtad, imagen, valor compartido, entre los estudiantes y egresados, se necesitan más estudios de mercadeo relacional en educación para comprender la relación con los docentes.

Por todo ello, se considera pertinente dar respuesta en este artículo al siguiente interrogante: ¿Cuáles son los modelos de mercadeo relacional que se han aplicado en educación y de qué manera involucran al docente universitario?, con el objetivo general de identificar los modelos de mercadeo relacional en la educación superior, se planteó una revisión sistemática de literatura de carácter heurístico, teniendo en cuenta a Tranfield y Smart (2003), con la planificación, conducción y construcción de informes.

Para lograr la comprensión y el desarrollo de la investigación, se hará una revisión de literatura de tipo exploratorio, indagando en las bases de datos Scopus y Google Scholar, donde se tendrán en cuenta los ejes principales de búsqueda: mercadeo educativo, modelos de mercadeo relacional y el docente en educación. Los criterios de inclusión fueron los artículos publicados en las revistas en temas de mercadeo 
y gestión educativa sin importar los tiempos de publicación.

En cuanto a los términos de búsqueda utilizados, fueron los siguientes, en inglés, por tratarse de la lengua con mayor difusión académica: corporate reputation, educational marketing, loyalty, relational marketing, satisfaction, teacher y trust Finalmente, a partir de los resultados se inicia un estudio exploratorio para la clasificación según los temas de pertinencia: universidad, mercadeo educativo, mercadeo relacional, docente, confianza, satisfacción, lealtad y reputación. Este tipo de investigación se realiza especialmente cuando el tema elegido ha sido poco explorado" (Sabino, 1992: 43)

\section{Gestión del mercadeo educativo}

La gestión del mercadeo en los entornos académicos se ha convertido en la herramienta que permite planear y consolidar estrategias con liderazgo que mejoren los indicadores de la universidad (Oplatka, Hemsley-Brown, 2007; Ramachandran 2010; Oplatka, 2018). Se han encontrado algunos modelos o esquemas de mercadeo que han sido aplicados en el sector educativo. A continuación, se muestra en el cuadro $N^{\circ} 1$ los principales objetivos que le dan el enfoque en cada uno de ellos:

\section{Cuadro 1 \\ Modelos de marketing educativo}

Autor

(Zeithaml, Parasuraman \& Malhotra, 2002); (Zeithaml, Parasuraman \& Berry, 1985)

(Bustos \& Becker, 2009)

(Diaz \& Sanabria, 2010)

(Larios, 2015)

(Sanz, 2017)

\section{Objetivo}

Proveer herramientas que permitan medir la calidad percibida, la cual a su vez permite segmentar el mercado en grupos de acuerdo con las expectativas que tienen acerca de un mismo servicio.

Convertir a los usuarios actuales, prospectos y potenciales en usuarios a largo plazo y así crear una posición educacional que sea atractiva al público y pueda mejorar las percepciones.

EI modelo MIGME permite, posibilitar el progreso de la oferta académica de las instituciones, de tal manera que se pueda ajustar a las necesidades de la sociedad y del sector productivo.

Identificar las características de la mezcla de mercadotecnia que realizan las Instituciones de Educación Superior, con el fin de proponer un nuevo modelo de mercadeo educativo.

Plantear estrategias de marketing a partir de los cinco pasos del CRM, lo cual permitirá dar una respuesta más determinada eficaz al cliente.

Fuente: Elaboración propia

Con el paso de los años, las organizaciones del sector educativo se han visto en la necesidad de implementar soluciones y estrategias para la prestación de sus servicios; por ello se plantea el marketing educativo como una opción indispensable. Manes (2000) lo define como un proceso de investigación de necesidades sociales a través de servicios educativos. De acuerdo a ello, autores como Bustos \& Becker (2009), Larios (2015), Diaz \& Sanabria 
Modelos de mercadeo racional en educación: articulación con el docente universitario Gómez-Bayona Ledy, Ferre-Pavia Carme, Arrubla Zapata Juan Pablo

(2010), Zeithaml, Parasuraman \& Malhotra (2002); Zeithaml, Parasuraman \& Berry, (1985) y Sanz, (2017), han planteado los anteriores modelos para la implementación del mercadeo de una manera más sencilla, dinámica y efectiva, llegando a la conclusión que la generación de estrategias de relación directa generan lealtad, recordación, bienestar y satisfacción en el cliente.

Teniendo en cuenta lo anterior, se considera necesario para este artículo analizar la importancia que trae para la gestión del mercadeo institucional, la articulación del docente como eje estratégico para generar valor en la apuesta del mercadeo relacional.

\section{Articulación del docente universitario con el mercadeo relacional}

Analizar al docente bajo la interpretación del mercadeo relacional es el desafío en esta investigación, por lo que se hace necesario comprender la gestión educativa y con ella las funciones del profesorado. La gestión educativa necesita ser repensada para que no se limite a ser administrada sino liderada con procesos pedagógicos, ya que la educación superior es uno de los medios más valiosos con los que se cuenta para lograr el desarrollo de la sociedad en entornos globalizados (López, 2008; Oplatka, 2018), y no solo en términos económicos, también en aspectos sociales que generen identidad nacional e internacional para contrarrestar las tendencias y desafíos que se presentan en América Latina (Enríquez, 2006), y en el mundo (Oplatka \& Hemsley-Brown, 2007).

De esta forma, las universidades necesitan establecer lineamientos para posicionarse adecuadamente en el mercado. Dorozhkin (2016) plantean como dentro de la gestión mercadológica, para visibilizar las organizaciones educativas, está el aprovechamiento de las capacidades al interior, como por ejemplo con los docentes, el relacionamiento al interior y exterior de la universidad y la comunicación asertiva. Así mismo, mencionan Mynbayeva y Yessenova (2016) que la imagen del docente se ha convertido en un atributo importante para la reputación, ya que se construye imagen pedagógica e imagen personal y estos aspectos construyen identidad, que se convierten en visibilidad para las organizaciones educativas.

De la misma manera, en la era de la sociedad del conocimiento, el docente debe repensarse (Martínez \& Ferraro, 2009), las capacidades y competencias deben influenciar positivamente, cumpliendo con altos estándares de calidad (González, 2011), ya que el estudiante espera recibir información cada día más completa; no solamente espera del docente la transferencia de conocimiento desde lo teórico, sino un conocimiento mayor desde lo práctico (Li, Granizo, \& Gardo 2016) y con técnicas de enseñanza diferentes, que permitan articular el conocimiento con otros estudiantes y hacer del trabajo en equipo una verdadera experiencia de aprendizaje (Emmett \& Robert 2005). Se espera que implementen metodologías de enseñanza de acuerdo con las nuevas tendencias tecnológicas para compartir el conocimiento (Brahimi \& Sarirete, 2015), y que finalmente, se encuentren satisfechos y motivados para que no deserten de la universidad (Zamora, Meza, \& Cox, 2018). 


\section{Modelos e importancia del mercadeo relacional en educación: principales hallazgos}

El primer punto de los resultados se centra en los 18 modelos de mercadeo relacional localizados en el cuadro 2, aplicados a la educación, los objetivos y las variables de estudio, que permitieron identificar la articulación con los públicos y especialmente, con el docente universitario.

Los resultados de la revisión de literatura también se enfocan a la importancia que las universidades le han venido dando al mercadeo educativo y la manera como han involucrado planes, estrategias y públicos para generar confianza y reputación entre sus stakeholders.

\subsection{Modelos de mercadeo relacional en el sector educativo}

Los modelos que se mostrarán en el cuadro $\mathrm{N}^{\circ} 2$, han permitido comprender los esquemas existentes de mercadeo relacional y la importancia que trae para el sector educativo la investigación del tema y la aplicabilidad en diferentes grupos de interés. De la misma manera, se han analizado los objetivos que contiene cada modelo y las características que han contrastado para validarlos teórica y empíricamente.

\section{Cuadro 2 \\ Modelos de mercadeo con enfoque relacional en educación}

\begin{tabular}{|c|c|c|}
\hline Autor & Objetivo del modelo & Propuesta de variable \\
\hline $\begin{array}{l}\text { (Hennig-Thurau, Langer, } \\
\text { \& Hansen , 2001) }\end{array}$ & $\begin{array}{l}\text { Propone a las instituciones educativas } \\
\text { asumir un rol más activo, lo que les } \\
\text { permitirá generar un nivel de participa- } \\
\text { ción más alto frente a temas como la } \\
\text { lealtad y las relaciones derivadas del } \\
\text { mercadeo relacional. }\end{array}$ & $\begin{array}{l}\text { Confianza. Calidad percibida. } \\
\text { Compromiso. Lealtad. Integración } \\
\text { en el sistema académico y social }\end{array}$ \\
\hline (Fernandez, 2011) & $\begin{array}{l}\text { Agente integrador de relaciones para } \\
\text { el fomento de la creación de valor per- } \\
\text { cibido por los egresados en el ámbito } \\
\text { universitario, logrando aplicar así las } \\
\text { directrices del mercadeo relacional en } \\
\text { la educación. }\end{array}$ & $\begin{array}{l}\text { Comunicación. Confianza. Satis- } \\
\text { facción. Conflicto funcional. Com- } \\
\text { promiso. Continuidad de expectati- } \\
\text { va. Voluntad. Colaboración }\end{array}$ \\
\hline $\begin{array}{l}\text { (Frasquet } \\
\text { \& Cervera, 2012) }\end{array}$ & $\begin{array}{l}\text { Comprobar que la confianza y la sa- } \\
\text { tisfacción tienen un grado alto de rela- } \\
\text { ción, aunque a veces estas se traten } \\
\text { por separada en un análisis de fondo } \\
\text { tienen cierta similitud y relación. }\end{array}$ & $\begin{array}{l}\text { Comunicación. Confianza. Satis- } \\
\text { facción. Conflicto funcional Com- } \\
\text { promiso. Continuidad de expecta- } \\
\text { tiva. Voluntad }\end{array}$ \\
\hline $\begin{array}{l}\text { (Schlesinger, Cervera, } \\
\text { \& Iniesta , 2012) }\end{array}$ & $\begin{array}{l}\text { Análisis mediante las relaciones y } \\
\text { entorno al marco de la formación a } \\
\text { lo largo de la vida del individuo en la } \\
\text { enseñanza superior y el desempeño } \\
\text { de la universidad en el nivel universi- } \\
\text { dad-egresado. }\end{array}$ & $\begin{array}{l}\text { Calidad interacción. Valores com- } \\
\text { partidos. Identificación. Compromi- } \\
\text { so. Lealtad }\end{array}$ \\
\hline
\end{tabular}




\section{Cont... Cuadro 2}

(Cervera, Schlesinger, Iniesta , \& Sánchez, 2012)

(Schlesinger, Cervera, Iniesta, \& Sánchez, 2014)

(Schlesinger, Cervera \& Calderón 2014)

(Schlesinger, Cervera, \& Iniesta, 2015)

(Liu \& Feng, 2016)

(Schlesinger, Cervera, \& Pérez-Cabañero, 2017)

(Chen, 2017)

(Asghar \& Pilkington 2018)

(Vallejo, Stefos, \& Narváez 2018)

(Alqirem, Husam \& Ahmed, 2018)

(Trullas, 2018)
Modelo para la medición de la imagen de la Universidad percibida por sus egresados, analizando que tanta influencia posee la imagen en la lealtad.

Interacción entre la universidad y el egresado, permitiendo mediante la conceptuación y medición de variables relativas, al comportamiento y relación con los empleados.

Analizar el papel de la confianza en la relación egresado-universidad, sus antecedentes y consecuencias.

Integración de conceptos que permita la respecta comprensión de la relación graduados universitarios

Conceptualización de las relaciones basadas en los efectos del liderazgo y cuál es el desempeño que este tiene fomentando la confianza de docentes y estudiantes.

Perspectiva de marketing relacional para investigar los roles de cuatro variables (imagen de marca, confianza, satisfacción y valores compartidos) de una manera directa e indirecta de la lealtad de los alumnos.

Explorar las relaciones estructurales entre las variables de mercadeo en el nivel superior del sector de la educación.

Encontrar las experiencias relacionadas a mercadeo y ejemplificar la interacción estudiante - docente

Porcentajes de satisfacción que perciben los estudiantes basándose en otros modelos que permiten evaluar la calidad de los servicios que prestan las instituciones.

Relación entre estudiantes universitarios que pueda reflejar una práctica adecuada dele importancia del mercadeo en el sector educativo

Modelo relacional basándose en antecedentes de los estudiantes, comenzando por la satisfacción, la capacidad corporativa, identificación del estudiante y la empleabilidad percibida, considerando principalmente el apoyo corporativo que percibe el estudiante por parte de la institución de educación.
Orientación. Capacitación. Reputación. Masificación. Accesibilidad. Imagen Universitaria. Identificación

Lealtad

Calidad. Interacción. Satisfacción. Imagen de universidad. Identificación Lealtad

Imagen. Confianza. Valores compartidos. Satisfacción. Lealtad. Valor percibido

Imagen. Calidad de la interacción Satisfacción. Identificación. Lealtad

Confianza. Liderazgo. Aprendizaje

Confianza. Imagen de la universidad. Satisfacción.

Lealtad. Valor compartido

Asociación de marca. Confianza estudiantil. Compromiso estudiantil. Satisfacción estudiantil.

Conocimiento. Valor. Acceso. Costo.

Servicios. Seguridad económica y emocional. Grupo de estudiantes Sistema de trabajo. Logros personales. Reconocimiento del éxito

Bonos sociales. Bonos financieros. Bonos estructurales

Bonos de relacionamiento. Satisfacción. Lealtad

Percepción de la calidad. Satisfacción. Identificación

Empleabilidad. Apoyo organizacional. Comunicación corporativa. Soporte organizacional. Relacionamiento

Promoción 


\section{Cont... Cuadro 2}

(Dzimińska, 2018)

(Zahoor, 2018)

(Berkovich, 2018)
Identificar el enfoque de cultura de la calidad de las universidades con la confianza como soporte estratégico.

Satisfacción y lealtad con identidad en la personalidad proactiva de los docentes

Identificar la frecuencia de perfiles de confianza de los docentes en el director, y las implicaciones de estos perfiles para el bienestar relacional de los docentes y sus comportamientos de ciudadanía organizacional (OCB).
Asociaciones de actitudes. Interacciones estudiantes-IES. Confianza. Cultura de calidad

Personalidad proactiva. Lealtad del estudiante. Satisfacción del estudiante. Compromiso de trabajo. Elaboración de trabajos.

Efecto positivo y negativo en las interacciones con el director.

Fuente: Elaboración propia.

Como resultado se evidencia que, al analizar los modelos o esquemas, básicamente cada uno presenta una contrastación de teorías que den respuesta a lo que se planteó como temas principales en las investigaciones, así mismo se evidencia que han sido probados en algunos grupos de interés como estudiantes y egresados. Así mismo que las variables que más se repiten en los modelos son confianza, satisfacción y lealtad, sintetizando que son estas tres variables las más representativas en mercadeo relacional.

De la misma manera se evidencia que el método que predomina para la recolección y análisis de datos es el uso de encuestas, que evalúan principalmente a los egresados de las instituciones, basándose en la lealtad y el compromiso que veían reflejados por parte de la institución, además de cuan satisfechos se sentían durante el proceso formativo. Como define Schlesinger (2012), de acuerdo con la implementación de estrategias, estas marcarán con cuanta probabilidad los estudiantes recomendarían la universidad a otras personas, persuadiéndolos de que estudien allí mismo.

También se encuentra que la comunicación para el manejo del marketing relacional ha sido un fuerte atributo aplicado a las instituciones de educación superior como herramienta que refuerza la imagen corporativa. Esta incide en lo que percibe un estudiante durante todo su proceso formativo, como mencionan Schlesinger, Cervera e Iniesta (2015). Específicamente preguntado a los exalumnos, fue el compromiso de la universidad, qué tanto apoyo sintieron de los docentes respectivos, y cuanta lealtad perciben en ellos, de manera que pudieran elegir el grado de recomendación que le dan a la institución. Esto arrojó conclusiones frente a la cercanía de la comunicación de la institución frente a los estudiantes.

Alineados a la satisfacción y la lealtad se sitúan Schlesinger, Cervera y Pérez Cabañero, tratando la variable de satisfaccion como la manera en que los ex alumnos se cuestionan sobre la elecccion que habían tomado 
Modelos de mercadeo racional en educación: articulación con el docente universitario Gómez-Bayona Ledy, Ferre-Pavia Carme, Arrubla Zapata Juan Pablo

al matricularse en un programa, evaluando el desarrollo del mismo y si fue una buena o mala eleccion según la percepcion del estudidante (Schlesinger, Cervera, \& Pérez Cabañero, 2017). En cuanto a la lealtad, cabe resaltar la gestion de transparencia orquestada por la institucion como parte de los factores que forjan una imagen. Finalmente los modelos de mercadeo relacional encontrados en el cuadro \# 2 llevan a la comprensión de un mercadeo holístico que articula las áreas de la comunidad académica y los diferentes grupos de interés, donde se considera que el docente es estratégico para abordar acciones que aportan significativamente en aspectos como la confianza, satisfacción, compromiso, valor compartido, construcción de marca, lealtad, entre otros. Siendo los docentes los que tienen un relacionamiento directo con los estudiantes y los encargados de construir empatía con quienes utilizan el servicio y tienen la alternativa de recomprar servicios a futuro.

\subsection{Estrategias y gestión mer- cadológica de las universida- des}

Los líderes educativos enfrentan grandes desafíos en la estructuración y consolidación de los modelos de gestión, ya que constantemente varían algunos aspectos culturales, sociales, políticos, económicos, donde se involucra el capital humano como eje de cualquier toma de decisión (Oplatka, Hemsley-Brown, 2007; Oplatka, 2018). Así, se hace necesario contextualizar los individuos que hacen parte de los entornos educativos y plantear alternativas que generen valor en las diferentes áreas que componen la organización (James \& Oplatka, 2018).

Para esta investigación, se identifica el área de mercadeo institucional como aquella encargada de planear y proyectar las necesidades de los diferentes públicos internos y externos a los que se enfoca la universidad. Esta área o departamento generalmente cuenta con la estructuración de planes o esquemas de mercadeo que son la ruta a seguir por un tiempo determinado y que está alineada a los valores misionales de la marca universitaria. Estos planes de mercadeo educativo son planteados desde las necesidades de cada región y país con el objetivo de ser pertinentes en la construcción de oferta académica y en la cobertura de programas de calidad (Arar, 2017).

Los sistemas de gestión en las estructuras educativas del mundo han permitido que se avance en la comprensión de los requerimientos de la sociedad, se han tratado de identificar las falencias del pasado y se ha reformulado la manera de hacer mercadeo en las organizaciones educativas. Aspectos como la globalización han hecho que los modelos académicos sean más cercanos a las necesidades de los individuos que cada día están más conectados a diferentes culturas y nuevas maneras de aprendizaje con componente de innovación y sostenibilidad (Oplatka, 2018); (Quiroga-Parra, 2018). Existen entonces herramientas de mercadeo que facilitan la gestión; una de ellas es la orientación al mercado desde los modelos de gestión educativo, que beneficia la proyección y los resultados de los directivos, ya que, al incorporarla dentro de la estructura funcional de las organizaciones académicas, se empieza a construir una cultura entre 
quienes hacen parte de la comunidad (Oplatka \& Hemsley, 2007). Ello permea los procesos administrativos y académicos para lograr mejores resultados en aspectos como la calidad del servicio, la comunicación estratégica entre los públicos y la identificación de necesidades reales de la sociedad para que sean abordados por la universidad.

James y Oplatka (2018) y Oplatka \& Hemsley (2007) mencionan que cuando los directivos de las universidades se adaptan a los requerimientos del medio, facilitan la manera de construir mercadeo institucional ya que van en sintonía con la articulación de los diferentes públicos internos y externos con los que se relaciona la universidad para generar identidad y proyectarla para darle posicionamiento y reconocimiento.

\subsection{El docente dentro del grupo de interés con mayor aporte a la gestión mercadológica}

Generalmente, las universidades cuentan con áreas de mercadeo o comunicación desde donde planean las estrategias para llegar a los públicos internos como estudiantes, administrativos, docentes, directivos 0 egresados. Asimismo, lo hacen a los públicos externos como proveedores, aliados, redes sociales y otros medios, sector estatal u oficial, empresarial y ciudadanía, entre otros. Las estrategias que desde ahí se gestan cuentan con objetivos e indicadores que apoyan el mercadeo institucional y proyectan la marca universitaria (Bennet, 2009). Menciona Akareem (2016) que la gestión del mercadeo en el entorno educativo se ha convertido en la oportunidad para identificar las fortalezas con las que cuenta el talento humano que forma parte de la universidad. Mynbayeva y Yessenova (2016) dan a conocer a los docentes como eje estratégico de la propuesta académica, siendo el mayor insumo con el que se cuenta para proyectar de manera intencional estrategias de mercadeo en el aula y fuera de ella. Algunas universidades han considerado este grupo de interés para construir identidad en la universidad, por eso les brindan alternativas que les permita estar satisfechos y así retenerlos. Se ha comprobado que de la motivación y la inspiración de los docentes será su productividad y la manera como ellos se empoderen de la marca universitaria (Akpayeva 2016).

$\begin{array}{cccc}\text { La gestión } & \text { académica } & \text { y } \\ \text { administrativa } & \text { que } & \text { realizan los } & \text { los } \\ \text { colaboradores en la universidad } & \text { es } \\ \text { representativa en la construcción } & \text { de }\end{array}$ identidad organizacional. Para consolidar este aspecto, las áreas de talento humano identifican alternativas como fortalezas y oportunidades del capital humano para proyectarlo y visibilizarlo en beneficio de la universidad (Varela \& Alvarez-Flores, 2019). Así, se han parametrizado algunos componentes de medición con el objetivo de conocer de qué manera se identifican debilidades en los colaboradores para brindar apoyo, al mismo tiempo se planean y ejecutan planes de clima organizacional que ayuden en la construcción de la cultura de la misma y del actuar responsablemente (Helguero \& Arrizabalaga, 2013).

Los docentes como integrantes activos de la universidad se benefician de las estrategias planteadas para generar satisfacción y que esto redunde en la retención. Aspectos como la claridad en las funciones sustantivas de la educación: docencia, investigación, proyección social e internacionalización, hacen que el docente cuente con una ruta de trabajo 
clara y que fortalezca sus competencias.

Es importante la comunicación del docente, que motive a la investigación y profundización de manera analítica (Oliver, 1998). Es por esto que el docente necesita motivación para enseñar, para transmitir en el aula el conocimiento de manera asertiva (Akpayeva, 2016). Poco se ha explorado de la importancia que trae para la gestión académica la satisfacción, el compromiso y lealtad de los docentes para lograr resultaos exitosos (Ting, 2011). Es necesario que las universidades tengan mayor compromiso con las estrategias de reclutamiento y retención de docentes que acompañe, motive e inspire a sus estudiantes (Bulger, Jones, Taliaferro, \& Wayda, 2015). El profesorado, como creador de relacionamiento, se adapta a la universidad y a los estudiantes para generar identidad y ser reconocido por los estudiantes y la comunidad (Wilkinson, Weiss, \& Grainger, 2013).

\section{Conclusiones}

En esta investigación, se han encontrado escasos modelos de mercadeo con enfoque relacional que permitan comprender las estrategias y la funcionalidad aplicadas en el campo de la educación. En cuanto a los modelos localizados, existen algunas posturas que muestran el enfoque de mercadeo relacional en educación a partir de algunos grupos de interés como egresados, estudiantes o la misma universidad, pero que poco se involucra al docente universitario en la propuesta de gestión mercadológica. Este es, sin duda, el aporte a esta investigación: la importancia de articular al docente de manera holística e intencional para que apoye en la construcción de mercadeo relacional con beneficio a la reputación corporativa de su institución.
Al mismo tiempo, se observa que las universidades vienen adaptando sus estrategias de gestión hacia acciones que le generen valor, para eso incorporan dentro de la planeación universitaria componentes mercadológicos que le brinden visibilidad y posicionamiento a la universidad. No es suficiente generar acciones de mercadeo, sino sensibilizar a los diferentes públicos con los que cuenta la universidad para construir una cultura de cooperación y lograr los objetivos de mercadeo bajo los lineamientos organizacionales.

Tanto como involucrar al docente de manera intencional dentro de la planeación de estrategias mercadológicas, resulta ser una importante apuesta para la gestión y el desarrollo de construcción en la satisfacción de los estudiantes, ellos, los docentes, son la parte principal en el sector educativo y por tanto son los influenciadores que necesita la universidad para motivar y mantener conectados a los estudiantes.

También se sintetiza que la construcción de confianza en los entornos académicos a partir del docente universitario naturalmente es la base para que los individuos perciban una buena comunicación y un oportuno relacionamiento para que logren una adecuada satisfacción que les brinde permanencia y motivación para que finalmente esto se convierta en lealtad. Solo la relación de los principales componentes de mercadeo relacional como son confianza, satisfacción y lealtad será la clave para que la reputación se construya al interior de la universidad y sea efectiva en los públicos tanto internos como externos.

Se ha venido avanzando en la producción investigativa en el tema de mercadeo con enfoque relacional 
en educación, pero continúa siendo poca para tenerla como referencia en Latinoamérica y especialmente en Colombia, es por esto que apostar a investigaciones de este tema es interesante para indagar que se está haciendo, cómo perciben el tema y cuáles son las estrategias que utilizan las instituciones para replicar modelos y mejorar los indicadores a nivel nacional e internacional. De esta manera, se aportará a la gestión mercadológica de las universidades y al buen desarrollo de estrategias que aporten oportunamente para el sostenimiento de las entidades de educación superior.

\section{Referencias Bibliográficas}

Akareem, Husain Salilul, and Syed Shahadat Hossain (2016), "Determinants of education quality: what makes students' perception different?" Open Review of Educational Research 3.1 52-67.

Akpayeva, Assel Bakirovna (2016), "Specifics of educational activity anti-motivation in future teachers subject to the training period."International Review of Management and Marketing" 6.3S, 265-269.

Alqirem, Raed Musbah, Husam Mustafa Alnaimi, and Ahmed Shuhaiber (2018), "A Best-Practice Model of University-Students Relationship Development: An Empirical Study." International Journal of Knowledge Management (IJKM) 14.4 46-67.

Amoli, Sara Javan, and Farnouche Aghashahi (2016), "An investigation on strategic management success factors in an educational complex." Procedia-Social and Behavioral Sciences 230 447-454.

Arar, Khalid (2017), "The characteristics of educational leadership in the
Middle East: A comparative analysis of three nation-states." The Wiley international handbook of educational leadership 355-373.

Asghar, Mandy, and Ruth Pilkington (2018), "The relational value of professional dialogue for academics pursuing HEA fellowship." International Journal for Academic Development 23.2 135-146.

Bennett, Roger, and Rehnuma Ali-Choudhury (2009), "Prospective students' perceptions of university brands: An empirical study." Journal of Marketing for Higher Education 19.1 85-107.

Berkovich, Izhak (2018), "Typology of trust relationships: Profiles of teachers' trust in principal and their implications." Teachers and Teaching 24.7 749-767.

Bulger, Sean (2015), "If you build it, they will come (or not): Going the distance in teacher candidate recruitment." Quest 67.1 73-92.

Bustos, H., and R. Becker (2009), "El libro lila del Marketing Educacional." Santiago de Chile: Publicación Digital.

Brahimi, Tayeb, and Akila Sarirete (2015), "Learning outside the classroom through MOOCs." Computers in Human Behavior 51 604-609.

Cervera, Amparo (2012), "Medición de la imagen de la universidad y sus efectos sobre la identificación y lealtad del egresado: una aproximación desde el modelo de Beerli y Díaz (2003)." Revista Española de Investigación de Marketing ESIC16.2 7-29.

Csikosova, Adriana, Mária Antošová, and Katarína Čulková (2014), "Strategy in direct and interactive marketing and integrated marketing communica- 
Modelos de mercadeo racional en educación: articulación con el docente universitario Gómez-Bayona Ledy, Ferre-Pavia Carme, Arrubla Zapata Juan Pablo

tions." Procedia-Social and Behavioral Sciences 116 1615-1619.

Chen, Yu-Chuan(2017), "The relationships between brand association, trust, commitment, and satisfaction of higher education institutions." International Journal of Educational Management 31.7 973-985.

Díaz, Milton Ricardo Ospina, and Pedro Emilio Sanabria Rangel (2010), "Un enfoque de mercadeo de servicios educativos para la gestión de las organizaciones de educación superior en Colombia: el modelo MIGME." Revista Facultad de Ciencias Económicas 18.2 107-136.

Dorozhkin, Evgenij M (2016), "The Experience of Positioning the University at the Educational Services Market." International Journal of Environmental and Science Education 11.16 9328-9338.

Dzimińska, Małgorzata, Justyna Fijałkowska, and Łukasz Sułkowski. (2018), "Trust-based quality culture conceptual model for higher education institutions." Sustainability 10.8 2599.

Fernández, Raquel Sánchez. (2011), "Modelo integrado de antecedentes y consecuencias del valor percibido por el egresado." Revista Venezolana de Gerencia 16.56 519-543.

Emmett, Dennis, and Robert Forget. (2005), "The utilization of activity-based cost accounting in hospitals." Journal of hospital marketing \& public relations 15.2 79-89.

Enríquez Clavero, José O. (2006), "Educación superior: tendencias y desafíos." Educación Médica 9.1 06-10.

Frasquet, Marta, Haydeé Calderón, and Amparo Cervera. (2012), "University-industry collaboration from a relationship marketing perspective:
An empirical analysis in a Spanish University." Higher Education 64.1 85-98.

González, Armando Lugo. (2011), "La calidad de la educación superior en Colombia." Revista científica 14 5671.

Helguero, María Matilde Schwalb, and Iñaki García Arrizabalaga. (2013), "Dimensiones de la responsabilidad social del marketing." Revista Venezolana de Gerencia 18.63 434-456.

Hennig-Thurau, Thorsten, Markus F. Langer, and Ursula Hansen. (2001), "Modeling and managing student loyalty: An approach based on the concept of relationship quality." Journal of service research 3.4 331-344.

James, Chris, Megan Crawford, and Izhar Oplatka. (2018), "An affective paradigm for educational leadership theory and practice: connecting affect, actions, power and influence." International Journal of Leadership in Education 1-12.

Kalenskaya, Nataliya, Ilshat Gafurov, and Aida Novenkova. (2013), "Marketing of educational services: Research on service providers satisfaction." Procedia economics and finance 5 368-376.

Larios-Gómez, Emigdio. (2015), "Mercadotecnia Educativa: Un Modelo Latinoamericano." Caderno Profissional de Marketing-UNIMEP3.1 14-33.

Li, Danni, Martina Gallarza Granizo, and Teresa Fayos Gardó. (2016), "The value trade-off in Higher Education service: A qualitative intercultural approach to students' perceptions." Intangible Capital 12.4 855-880.

Liu, Shengnan, Philip Hallinger, and Daming Feng. (2016), "Learning-cente- 
red leadership and teacher learning in China: Does trust matter?." Journal of Educational Administration 54.6 661-682.

López Segrera, Francisco. (2008), "Tendencias de la educación superior en el mundo y en América Latina y el Caribe." Avaliação: Revista da Avaliação da Educação Superior (Campinas) 13.2 267-291.

Manes, Juan Manuel. (2000), "Marketing educativo, gestión estratégica y calidad total: un círculo virtuoso." Organización y Gestión educativa 5 3-8.

Martínez, Alicia, and Ana Ferraro. (2009), “El profesor universitario. Reflexiones acerca de la esencia del docente universitario en la sociedad actual." Argentina: Universidad Tecnológica Nacional. Recuperado en: http://www. edutecne. utn. edu. ar/debates/el_profesor_universitario. pdf (29/05/2014).

Mynbayeva, Aigerim, and Kamchat Yessenova. (2016), "Distinguishing Features of Teacher Image and Faculty Member's Image: Comparative Study." International Review of Management and Marketing 6.3S 110-117.

Oliver, Sandra M. (1998), "Technology assisted teaching and learning: Design implications for communication courses on the internet." Journal of Communication Management 3.1 26-38.

Oplatka, Izhar. (2018), “Understanding Emotion in Educational and Service Organizations through Semi-Structured Interviews: Some Conceptual and Practical Insights." The Qualitative Report 23.6 1347-1363.

Oplatka, Izhar, and Jane HemsleyBrown. (2007), "The incorporation of market orientation in the school culture: An essential aspect of school marketing." International Journal of Educational Management 21.4 292-305.

Oplatka, Izhar, and Ben Gurion. (2006), "Teachers' perceptions of their role in educational marketing: Insights from the case of Edmonton, Alberta." Canadian Journal of Educational Administration and Policy 51.

Osayawe Ehigie, Benjamin, and Megan Taylor. (2009), "Managing students' loyalty to school after graduation through relationship marketing." The TQM Journal 21.5 502-516.

Ramachandran, Narayanan T. (2010), "Marketing framework in higher education: Addressing aspirations of students beyond conventional tenets of selling products." International Journal of Educational Management 24.6 544-556.

Rojas, Javier Mendoza. (2018), "Situación y retos de la cobertura del sistema educativo nacional." Perfiles Educativos 40.Especial 11-52.

Quiroga-Parra, Darío Jesús.b(2018), “Innovación en México y Colombia: un análisis comparado teórico y empírico." Revista Venezolana de Gerencia 23.85 .

Sabino, Carlos. (1992), "El proceso de investigación, Ed." Panapo, Caracas .

Sanz, Daniel. (2017), "Marketing educativo como estrategia de negocio en universidades privadas." Desarrollo Gerencial 9.1 160-175.

Schlesinger, W., A. Cervera, and $\mathrm{H}$. Calderón. (2014), "El papel de la confianza, la imagen y los valores compartidos en la creación de valor y lealtad: aplicación a la relación egresado-universidad." Revista Española de Investigación de Marketing ESIC 18.2 126-139. 
Modelos de mercadeo racional en educación: articulación con el docente universitario Gómez-Bayona Ledy, Ferre-Pavia Carme, Arrubla Zapata Juan Pablo

Schlesinger, Walesska, Amparo Cervera, and M. Ángeles Iniesta. (2015), "Key elements in building relationships in the higher education services context." Journal of Promotion Management 21.4 475-491.

Schlesinger, Walesska. (2012), "La identificación egresado-universidad: un modelo desde un enfoque de marketing relacional." Revista de Ciencias Sociales 18.1

Schlesinger, María Walesska. (2014), "Un enfoque de marketing de relaciones a la educación como un servicio: aplicación a la Universidad de Valencia." INNOVAR. Revista de Ciencias Administrativas y Sociales 24.53 113-125.

Schlesinger, Walesska, Amparo Cervera, and Carmen Pérez-Cabañero. (2017), "Sticking with your university: The importance of satisfaction, trust, image, and shared values." Studies in Higher Education 42.12 21782194.

Ting, Shueh-Chin. (2011), "The effect of internal marketing on organizational commitment: job involvement and job satisfaction as mediators." Educational Administration Quarterly 47.2 353-382.

Tranfield, David, David Denyer, and Palminder Smart. (2003), "Towards a methodology for developing evidence $\square$ informed management knowledge by means of systematic review." British journal of management 14.3 207-222.

Trullas, Irene. (2018), "Student-perceived organizational support and perceived employability in the marketing of higher education." Journal of Mar- keting for Higher Education 28.2 266-281.

Varela, Rosa Elena Richart, Erika P. Álvarez-Flores, and Reyna Del Carmen Martínez-Rodríguez. (2019), "Competencias del perfil del administrador." Perfiles Educativos 41.164 82-98.

Vallejo, Adriana, Efstathios Stefos, and Adrián Narváez. (2018), "Service quality analysis of universities in cuenca, ecuador." International Journal for Quality Research 12.1.

Wilkinson, Lydia, Peter Eliot Weiss, and Raj Grainger. (2013),"MyCl: Crossing the Border of student and communication instructor relationships." IEEE International Professonal Communication 2013 Conference. IEEE.

Zahoor, Adil. (2018), "Teacher Proactivity Influencing Student Satisfaction and Loyalty Role of Job Crafting and Work Engagement." Vikalpa 43.3 125-138.

Zamora Poblete, Guillermo Manuel, Marisa Meza Pardo, and Pilar Cox Vial. (2018), "Profesores principiantes de educación secundaria en Chile. Pese a las dificultades, $¿$ qué los hace permanecer como docentes?." Perfiles educativos 40.160 29-46.

Zeithaml, Valarie A., Arun Parasuraman, and Arvind Malhotra. (2002), "Service quality delivery through web sites: a critical review of extant knowledge." Journal of the academy of marketing science 30.4 362-375.

Zeithaml, Valarie A., Ananthanarayanan Parasuraman, and Leonard L. Berry. (1985), "Problems and strategies in services marketing." Journal of marketing 49.2 33-46.

- Esta obra está bajo una licencia de Creative Commons Reconocimiento-NoComercialCompartirlgual 3.0 Unported. http://creativecommons.org/licenses/by-nc-sa/3.0/deed.es_ES 